\section{S15 IS CONTRAST-ENHANCED COMPUTED TOMOGRAPHY (CT) SCORING A USEFUL TOOL IN PREDICTING PLEURAL MALIGNANCY ON THORACOSCOPY?}

doi:10.1136/thoraxjnl-2012-202678.021

${ }^{1} \mathrm{~B}$ Buckley, ${ }^{2} \mathrm{M}$ Haris, ${ }^{2} \mathrm{~B}$ Miller, ${ }^{2} \mathrm{~S}$ Leyakathali khan, ${ }^{2} \mathrm{~S}$ Diver, ${ }^{2} \mathrm{~S}$ Kearney, ${ }^{2} \mathrm{M}$ Munavvar. ${ }^{1}$ School Of Medicine, Universtiy of Manchester, Manchester, UK; ${ }^{2}$ Royal Preston Hospital, Preston, UK

Background CT and Thoracoscopy are useful tools in the evaluation of patients with pleural diseases. Previous studies have shown that certain features on CT may help in differentiating malignant and benign pleura and these may correlate with thoracoscopic findings. We aimed to assess whether a simple scoring system based on CT appearance criteria could be used to predict the likelihood of pleural malignancy.

Methods 104 patients who underwent semi-rigid thoracoscopy between January 2008 and April 2012 were studied. The scans were reviewed by the Chest Radiologist who was blinded to the thoracoscopic findings and pleural biopsy results. Five CT features were given a score of $1-3$, where 3 suggested a feature was present, 1 not present and 2 if uncertain. An overall impression of likelihood of malignancy was then scored 1-3, 3 being malignant, 1 benign and 2 indeterminate. Data was analysed using chi-square and logistic regression.

Results $\mathrm{N}=104$, mean age 74 years (range 40-93); female 35(34\%), male 69(66\%). Pleural biopsy histology was available in 90 of which $57(63 \%)$ were malignant and $33(37 \%)$ benign; 7 did not have biopsy and 7 were either insufficient or non-diagnostic. In those with malignancy, pleural thickening was noted in 43 (sensitivity $88 \%$, specificity $34 \%$ ) and nodularity in 29 (sensitivity $52 \%$, specificity $82 \%$ ) on thoracoscopy.

Using a score of 1 and 3, specificity and sensitivity for circumferential/diffuse pleural thickening was $39 \%$ and $65 \%$, nodular pleural thickening $94 \%$ and $53 \%$, parietal pleural thickening $>1 \mathrm{~cm} 94 \%$ and $26 \%$, mediastinal involvement $76 \%$ and $63 \%$, smooth pleural thickening $33 \%$ and $81 \%$, and overall impression score was $84 \%$ and $63 \%$. Total score $\geq 8$ for all 5 features and the overall impression $(p<0.0001)$ was significantly associated with malignancy.

A score of 3 for nodular thickening $(p<0.0001)$, parietal thickening $(p=0.03)$, mediastinal involvement $(p=0.0011)$ and a combined score $\geq 4$ for these 3 features (sensitivity $79 \%$, specificity $73 \%, p=0.0001$ ) was highly suggestive of malignancy.

Conclusion These results suggest that a CT scoring system based on the above features can be reliably used to predict pleural malignancy prompting more intensive evaluation such as thoracoscopy, although this will require further validation.

Abstract S15 Table 1 CT prediction score comparing Malignant and Benign pleura

\begin{tabular}{lcl}
\hline $\begin{array}{l}\text { CT appearance and score } \\
\text { Diffuse Pleural thickening }\end{array}$ & $\begin{array}{c}\text { Malignant pleura } \\
(\mathbf{n}=\mathbf{5 7})\end{array}$ & $\begin{array}{c}\text { Benign pleura } \\
(\mathbf{n}=\mathbf{3 3})\end{array}$ \\
\hline 1-Low & $20(35 \%)$ & $13(39 \%)$ \\
2-Moderate & 0 & 0 \\
3-High & $37(65 \%)$ & $20(61 \%)$ \\
Nodular Pleural thickening & & $31(94 \%)$ \\
1- Low & $27(47 \%)$ & $1(3 \%)$ \\
2- Moderate & $3(5 \%)$ & $1(3 \%)$ \\
3- High & $27(47 \%)$ & $31(94 \%)$ \\
Parietal Pleural thickening & & 0 \\
1- Low & $42(74 \%)$ & $2(6 \%)$ \\
2- Moderate & 0 &
\end{tabular}

\begin{tabular}{lll}
\hline 3- High & & \\
Mediastinal involvement & & \\
1- Low & $21(37 \%)$ & $2(6 \%)$ \\
2- Moderate & $7(12 \%)$ & $6(18 \%)$ \\
3- High & $29(51 \%)$ & \\
Smooth Pleural thickening & & $11(33 \%)$ \\
1- Low & $11(19 \%)$ & 0 \\
2- Moderate & $1(2 \%)$ & $22(67 \%)$ \\
3- High & $45(79 \%)$ & 0 \\
Overall likelihood of malignancy on & & $28(85 \%)$ \\
CT Pleural scoring & & $5(15 \%)$ \\
1- Low & 0 & \\
2- Moderate & $21(37 \%)$ & \\
3- High & $36(63 \%)$ & \\
\hline
\end{tabular}

Categorical variables shown as $\mathrm{N}(\%)$

\section{S16 A LARGE, PROSPECTIVE, MULTICENTRE STUDY EVALUATING THE SURVIVAL OF PATIENTS WITH MALIGNANT PLEURAL EFFUSION ACCORDING TO THE UNDERLYING CELL TYPE}

doi:10.1136/thoraxjnl-2012-202678.022

${ }^{1} \mathrm{AO}$ Clive, ${ }^{2} \mathrm{CE}$ Hooper, ${ }^{3} \mathrm{ETH}$ Fysh,,${ }^{4} \mathrm{C}$ Tobin, ${ }^{2} \mathrm{AJ}$ Morley, ${ }^{2} \mathrm{~N}$ Zahan, ${ }^{2} \mathrm{C}$ Sellar, ${ }^{1} \mathrm{R}$ Bhatnagar, ${ }^{2}$ AR Medford, ${ }^{3} Y C G$ Lee, ${ }^{1} N A$ Maskell. 'University of Bristol, Bristol, UK; ${ }^{2}$ North Bristol NHS Trust, Bristol, UK; ${ }^{3}$ University of Western Australia, Perth, Western Australia; ${ }^{4}$ Sir Charles Gardiner Hospital, Perth, Western Australia

Malignant pleural effusion (MPE) is a common clinical problem, which causes significant morbidity and has a variable prognosis. This is the largest series to date evaluating the survival of patients with MPE according to their underlying cell type.

Methods We prospectively collected data on patients presenting with MPE from two large pleural services over a 3 year period. All patients gave written informed consent. Patients were followed up for a minimum of 9 months or until death or loss to follow up (whichever was sooner). For survival analysis, the log rank (MantelCox) test was applied to Kaplan Meier survival curves.

Results Data was collected on 466 patients. The median age of the patients was 71 (IOR 65-79) and 63\% were male. 56\% of effusions were right sided and $41 \%$ patients had an effusion occupying $>50 \%$ of the hemithorax. $73 \%$ of patients had confirmation of pleural malignancy based on cytology or pleural biopsy, 21\% had a presumed malignant effusion with confirmed malignancy elsewhere and $6 \%$ had a radiological diagnosis.

Patients with a pleural effusion secondary to mesothelioma $(n=148)$ had the longest median survival (MS) at 339 days. This is significantly longer than those effusions caused by lung cancer ( $\mathrm{n}=$ 127, MS 71 days) (95\% CI $-0.56-4.45, p<0.0001)$. Cell types with an intermediate survival, included gynaecological malignancy ( $n=33 ; M S=268$ days), urological cancer $(n=11 ; M S=220$ days), gastrointestinal malignancy ( $\mathrm{n}=16$; MS 268 days), haematological malignancy ( $\mathrm{n}=27$; median survival 204 days) and breast cancer ( $n=48 ;$ MS 204 days). Lung cancer, adenocarcinoma of unknown primary ( $\mathrm{n}=9$; MS 87 days) and sarcoma ( $\mathrm{n}=6$, MS 65.5 days) had the shortest survival.

The Kaplan-Meier survival curve for the 3 largest groups is shown in the figure.

Conclusions Selecting the most appropriate strategy for management of malignant pleural effusion depends on patient choice, their clinical condition and the perceived prognosis. This data confirms that the survival of patients with malignant pleural effusion varies widely depending on the site of their primary malignancy. Accounting for this may help to better inform patients of their prognosis and aid clinical decision making. 